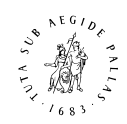

\title{
The Redundant Signals Effect and the Full Body Illusion: not Multisensory, but Unisensory Tactile Stimuli Are Affected by the Illusion
}

\author{
Lieke M. J. Swinkels, Harm Veling and Hein T. van Schie * \\ Behavioural Science Institute, Radboud University, Montessorilaan 3, 6500 HR Nijmegen, \\ The Netherlands
}

Received 1 September 2020; accepted 28 February 2021

\begin{abstract}
During a full body illusion (FBI), participants experience a change in self-location towards a body that they see in front of them from a third-person perspective and experience touch to originate from this body. Multisensory integration is thought to underlie this illusion. In the present study we tested the redundant signals effect (RSE) as a new objective measure of the illusion that was designed to directly tap into the multisensory integration underlying the illusion. The illusion was induced by an experimenter who stroked and tapped the participant's shoulder and underarm, while participants perceived the touch on the virtual body in front of them via a head-mounted display. Participants performed a speeded detection task, responding to visual stimuli on the virtual body, to tactile stimuli on the real body and to combined (multisensory) visual and tactile stimuli. Analysis of the RSE with a race model inequality test indicated that multisensory integration took place in both the synchronous and the asynchronous condition. This surprising finding suggests that simultaneous bodily stimuli from different (visual and tactile) modalities will be transiently integrated into a multisensory representation even when no illusion is induced. Furthermore, this finding suggests that the RSE is not a suitable objective measure of body illusions. Interestingly however, responses to the unisensory tactile stimuli in the speeded detection task were found to be slower and had a larger variance in the asynchronous condition than in the synchronous condition. The implications of this finding for the literature on body representations are discussed.
\end{abstract}

\section{Keywords}

full body illusion, multisensory integration, redundant signals effect, race model, cross-modal

\footnotetext{
* To whom correspondence should be addressed. E-mail: h.vanschie@bsi.ru.nl
} 


\section{Introduction}

The full body illusion (FBI), is a perceptual illusion in which participants experience a change in self-location towards a body that they see in front of them from a third-person perspective and in which they experience touch to originate from this body (Lenggenhager et al., 2007). To induce the illusion, the participant wears a head-mounted display (HMD) that shows a live feed of a connected camera that is positioned approximately $1.5 \mathrm{~m}$ behind the participant. The FBI can be induced by an experimenter who provides synchronous visual and tactile stimulation by stroking the participants on the back (Aspell et al., 2009; Lenggenhager et al., 2007), by means of a robotic stroking device (Salomon et al., 2013) or by the participants themselves using a robotic stroking device (Hara et al., 2014), neck stroking (Swinkels et al., 2020a) or stroking movements without touch (Swinkels et al., 2020b). Multisensory integration of synchronous signals originating from the different modalities is thought to underlie the illusion (Botvinick and Cohen, 1998; Ehrsson, 2012; Ehrsson et al., 2005). Since the first description of the FBI and the related rubber-hand illusion (RHI; Botvinick and Cohen, 1998) in the literature, researchers have continuously looked for new measures to establish whether an illusion occurred. Many subjective and more objective measures have been introduced. However, these measures have not always led to clear and unambiguous results in the past and some may not measure what they intend to measure. Researchers also seem to disagree on the best subjective measure to establish whether an illusion occurred. In the present paper we will provide an overview of some of the measures that are often used and potential issues related to them and test a new objective measure of the illusion that was designed to tap directly into the multisensory integration underlying the illusion. We believe that the field would benefit from a new objective measure that circumvents existing issues because it can aid in establishing whether an illusion occurred or not in a more reliable manner. Ultimately this may help us in gaining insight in the mechanisms that are responsible for our embodied bodily experiences in general and the potential transfer of embodiment to virtual bodies.

\subsection{Illusion Questionnaires}

A common way to assess the presence of an illusion is to ask participants to complete a questionnaire following a session of synchronous or asynchronous stimulation (Aimola Davies et al., 2013). Participants are usually asked questions that tap into the transfer of touch towards the virtual body, self-location, causality and body ownership (e.g., Aimola Davies et al., 2013; Botvinick and Cohen, 1998; Lenggenhager et al., 2007; Swinkels et al., 2020a). 
Despite their widespread use, there are some problems related to the use of illusion questionnaires. Questionnaires are subjective in nature and can provide a wealth of information if one is interested in the subjective nature of the illusion but at the same time this subjective nature can make it difficult to quantify the illusion; for instance, problems with anchoring may arise that leave the experimenter in the dark as to what a participant would consider a strong illusion on a certain scale or how a participant uses the scale. Do participants consider a score of 40 as an illusion experience on a scale that ranges from 0 (not at all) to 100 (very strongly) or only when they indicate 90? Do participants use the 0 on a -3 to +3 scale to indicate they did not have an experience, to indicate that they do not know, or to indicate that they neither agree nor disagree with a statement (Lane et al., 2017)? Apart from the ambiguity that exists in the interpretation of scores in subjective scales, individual differences in the interpretation of scale points and anchors may be especially problematic in between-subject designs where variations in interpretation may add considerable amounts of unexplained variance, and might even contribute to false positives in small samples. This problem is circumvented in withinsubject designs, as participants may be expected to apply the same subjective use of a scale in either condition. An alternative way to deal with individual differences in the interpretation of scales is to use an ipsatization procedure which centres scores relative to the participant average score and standard deviation across conditions (Romano et al., 2014; Schwartz, 1992; Tieri et al., 2015; Tosi et al., 2020). However, the use of within-subject designs and ipsatization do not provide a solution to the problem of how to interpret subjective scores and in determining whether a body illusion was induced or not.

Another - more general - problem with questionnaires is that questions are often open to interpretation, so participants may interpret them differently than how they were intended by the experimenter (Schwarz and Oyserman, 2001). The wording of the illusion statements tends to be complex and previous research from our own lab suggests that participants may find it hard to capture their experiences in words when asked about them. Participants may also find it difficult to link their experiences to the statements in the questionnaire, resulting in different interpretations of the questions than intended by the experimenter (Swinkels et al., 2020b). This observation is in line with the idea that embodiment is rich and complex in nature but, at the same time, also elusive and hard to describe (Gallagher, 2006; Haggard and Wolpert, 2005; Longo et al., 2008). Additionally, there may be problems with social desirability (i.e., participants responding with what they think the experimenters want to hear) and experimenter demand effects (i.e., changes in behaviour in the experiment due to cues that constitute the 'correct' behaviour in the setting; Zizzo, 2010). Finally, but not unimportantly, there seems to be little agreement at present on how the illusion should be measured. Not only do experimenters use different 
scales, there also seems to be little agreement about which questions measure the illusion and which measure experimenter demand (e.g., Botvinick and Cohen, 1998; Kalckert and Ehrsson, 2012; Kokkinara and Slater, 2014; Lenggenhager et al., 2007; Maselli and Slater, 2013; Thür et al., 2019).

Some studies use interviews or free recall in addition to illusion questionnaires to obtain a better insight into the illusion experienced by participants (Altschuler and Ramachandran, 2007; Longo et al., 2008; Swinkels et al., 2020b). Even though participants find it hard to capture their experiences in words when asked about them, previous research in our lab suggests that interviewing the participants may partially resolve some of the problems with questionnaires (Swinkels et al., 2020b). More specifically, in addition to obtaining insight in different characteristics of the illusion and specific experiences participants may have had, interviews may shed light on the interpretation of the questions and the usage of the scale and may in that way help to elucidate whether an illusion was experienced or not (see Note 1).

\subsection{Proprioceptive Drift}

Used alongside illusion questionnaires, researchers have measured proprioceptive drift which has been argued to provide a more objective measure of the illusion that may be less prone to subjective interpretation. Similar to questionnaires, proprioceptive drift can only be measured after the experimental session and not while the stimulation is ongoing. Proprioceptive drift is measured by asking blindfolded participants to point to the felt location of the hand that is involved in the illusion (RHI; Botvinick and Cohen, 1998). Studies have found that participants tended to overshoot the real location of their hand in the direction of the illusory hand. This displacement has been found to be significantly correlated with the strength of the illusion (Abdulkarim and Ehrsson, 2016; Botvinick and Cohen, 1998; Kalckert and Ehrsson, 2012, 2014; Tsakiris et al., 2005). A similar procedure has been conceived for the FBI. Participants are displaced backwards and are asked to walk back to the location where they felt their body to be before (Lenggenhager et al., 2007). As with the RHI participants overshoot the real location of their body in the direction of the illusory body. However, as participants could rely on the proprioceptive memory of the steps they had taken while moving backwards it is unclear whether this task measures the experience of the FBI in a valid way.

Some additional concerns need to be mentioned as well. We believe that it stands to debate whether the proprioceptive task truly solves problems with experimenter demand and social desirability, as proprioceptive drift measures typically require direct contact between the participant and the experimenter which could (unconsciously and unwillingly) drive or influence responses by the participant. The task is still subjective in the sense that the measure of proprioceptive drift asks participants to estimate the position of their hand. 
However, this task may be preferable as it is at least more straightforward and easier to understand than some of the illusion questions.

Furthermore, some researchers question the usefulness of proprioceptive drift as a measure of the illusion due to mixed findings with this paradigm. Some studies reported more proprioceptive drift in the illusion condition, but did not obtain a significant correlation between proprioceptive drift and perceived ownership (Metral et al., 2017; Riemer et al., 2015, 2019). Raz et al. (2020) found the correlation between proprioceptive drift and questionnaire scores in some conditions, but not in others. Most strikingly, Rohde et al. (2011) and Holle et al. (2011) found increased proprioceptive drift in the absence of the illusion, which suggests that factors other than the illusion may influence this measure.

\subsection{Physiological Responses to Threat}

Another way to measure the illusion is by collecting physiological responses when a stimulus is presented that threatens the illusory body(part). This method has mainly been used with the RHI and the full body ownership illusion in virtual reality, whereby a fake or virtual body part can easily be threatened without any chance of actual physical harm for the participant. When participants experience the illusory body(part) as their own, skin conductance response (SCR) has been found to increase when the illusory body (Ehrsson, 2007; Ferri et al., 2013; Guterstam et al., 2015; Petkova and Ehrsson, 2008; Petkova et al., 2011; Preston et al., 2015; Preuss and Ehrsson, 2019; Tacikowski et al., 2020a, b; van der Hoort et al., 2011) or body part is threatened (Armel and Ramachandran, 2003; Gentile et al., 2013; Guterstam et al., 2013; Petkova and Ehrsson, 2009; Samad et al., 2015). A similar result has been obtained for heart rate deceleration (HRD; Pomés and Slater, 2013; Slater et al., 2010). SCR and HRD in response to threat are typically found to be stronger in the illusion condition than in the control condition in which the body(part) is not considered as one's own. An advantage of both the SCR and the HRD is that these measures are objective in the sense that participants cannot control these responses (Ma and Hommel, 2013) and that these measures can be measured during the illusion. However, neither of the two physiological measures can be recorded continuously, as the threat that is evoking the physiological response becomes predictable and is less threatening when the threat is presented repeatedly. As a consequence, SCR and HRD to threatening stimuli may be difficult to apply in a within-subject context. Furthermore, as mentioned earlier, the use of physiological responses to threat is especially useful with paradigms in which the actual body of the participant remains safe from harm when threatened, which is not the case with the FBI paradigm. 


\subsection{The Cross-Modal Congruency Effect (CCE)}

A promising measure that is used to directly tap into the change in self-location during the illusion is the cross-modal congruency task or CCT (e.g., Aspell et al., 2009; Zopf et al., 2010) that measures the cross-modal congruency effect (CCE; Pavani et al., 2000; Spence et al., 2004). In this speeded discrimination task, participants respond to the location (up vs down) of tactile stimuli presented to their left or right hands (RHI) or to the left or right side of their back (FBI) while ignoring visual distracters that can be presented at each of these four spatial locations, i.e., congruent (same elevation) and incongruent (different elevation) locations on the same and on the opposite side of the body. The difference in reaction time between congruent and incongruent trials is called the CCE and has been argued to reflect the amount of multisensory integration between tactile targets and visual distracters (Zopf et al., 2010). The CCE is typically found to be larger when distractors are presented on the same side of the body, as compared to the opposite side of the body. The illusion is thought to result in an integration of visual and tactile stimulation at the location of the virtual body (Aspell et al., 2009; Maselli and Slater, 2014) Various experiments have demonstrated that body illusions indeed modulate the CCE, with a larger CCE in the synchronous condition where an illusion is experienced compared to the asynchronous condition where no illusion is experienced (Aspell et al., 2009; Maselli and Slater, 2014; Rognini et al., 2013; Zopf et al., 2010).

However, two confounding explanations have been provided for the CCE that may limit the use of the CCT as a measure of multisensory integration in body illusions (Forster et al., 2008; Marini et al., 2017; Spence et al., 2004). First, the CCE is found to (partly) reflect response competition that occurs when visual distractors are presented at incongruent (relative to congruent) locations and prime the opposite (relative to the same) response (Spence et al., 2004). Second, the CCE is found to (partly) reflect exogenous cueing effects whereby the visual distractor attracts attention, resulting in a facilitation of reaction times if the tactile stimulus happens to be presented in the same location and in a hampering of the reaction times if the tactile stimulus is presented in the opposite location. Although a small part of the CCE effect may actually reflect multisensory integration between visual and tactile stimuli (Forster $e t$ al., 2008; Marini et al., 2017; Spence et al., 2004), the CCT does not seem suitable to provide an unambiguous measure of multisensory integration that can be used to determine the presence of an illusion (Marini et al., 2017; Patané et al., 2019). 


\subsection{Testing a New Objective Measure of the Illusion: the Redundant Signals Effect}

A measure that does not suffer from response conflict or exogenous attention confounds and that is often used to measure multisensory integration in the broader literature is the redundant signals effect (RSE). This effect occurs when participants are asked to make speeded responses to the onset of any stimulus and reflects the relative gain in reaction time (RT) when stimuli are presented simultaneously in multiple modalities, as compared to when a stimulus is presented in a single modality (Minakata and Gondan, 2018; Schröter et al., 2009). This effect has been demonstrated with various stimulus types and can be explained in multiple ways. The two most frequently used explanations are the statistical facilitation account (Raab, 1962) and the coactivation account (Miller, 1982) of which multisensory integration is an example (Brandwein et al., 2010). When statistical facilitation is driving the effect, the relative speeding up of reaction times on the multimodal trials is driven by the stimulus that is processed the fastest (Gondan, 2010; Raab, 1962). This process is described in the form of a race model (Miller, 1982). In coactivation models (Miller, 1982) on the other hand, both stimuli jointly provide activation to satisfy the response criterion. As activation builds up faster when it is provided by two sources, participants respond faster to the multisensory stimuli than to the unisensory stimuli. Miller's race model inequality test can be used to determine what is causing this relative 'speeding up' on the multisensory trials (Miller, 1982, 1986). If the test indicates a violation of the race model, the speeding up can be explained by a coactivation account; if the test indicates that the race model was not violated, the speeding up can be explained by statistical facilitation.

In the present paper, we tested whether the redundant signals effect (RSE) would be differentially affected by the FBI. We presented a visual stimulus on the participant's virtual body and a vibrotactile stimulus on the participant's real body. To create a multisensory stimulus these stimuli could also be presented simultaneously. During a FBI, both visual stimulation that is presented on the virtual body and tactile stimulation that is presented on the real body are experienced at the location of the virtual body (e.g., Lenggenhager et al., 2007; Swinkels et al., 2020b). This is not the case when no illusion is induced; in the asynchronous condition visual and tactile stimuli are typically experienced at separate locations, i.e., at the location of the virtual body and at the real body respectively. In a similar vein, and in line with the CCE literature, we expected the illusion to result in an integration of the visual and vibrotactile stimulus of the RSE task when they are presented simultaneously (Maselli and Slater, 2014), with participants experiencing both the virtual and vibrotactile stimulation as if they originate from the virtual body. In the asynchronous 
control condition where no illusion is induced participants should experience the stimulation as occurring simultaneously but on two different bodies, the visual stimulation should be experienced on the virtual body and the vibrotactile stimulation should be experienced on the real body. However, following the statistical facilitation account, participants may still respond faster to the multimodal stimuli compared to the unimodal stimuli but there should be no support for the integration account.

In sum, in the present paper we hypothesised that: (1) a FBI is evoked in the experimental synchronous condition but not in the asynchronous control condition; (2) the processing of multisensory stimuli in the synchronous experimental condition demonstrates multisensory integration in accordance with a coactivation model; and (3) the processing of multisensory stimuli in the asynchronous control condition is influenced by statistical facilitation but not by multisensory integration. The following predictions follow from these hypotheses: (1) we expected the scores on illusion statements S1-S3 to be higher in the synchronous as compared to the asynchronous condition (see Table 1); (2) we expected faster RTs to the multisensory trials than to the unisensory trials in the synchronous condition. We expected multisensory integration of the multisensory stimuli to occur in the synchronous condition, which should be evidenced by a violation of the race model; and (3) we similarly expected faster RTs to the multisensory trials than to the unisensory trials in the asynchronous condition. Importantly, we expected no violation of the race model in the asynchronous condition, indicating that this speeding

\section{Table 1.}

Illusion statements that were answered on a scale ranging from 0 (not at all) to 100 (very strongly) unless indicated otherwise

Item Statement

Please indicate to what extent...

S1 ...you felt the touch of the brush at the location where you saw the virtual body being touched

S2 ... the touch you felt was caused by the brush touching the virtual body

S3 ... you felt your body was located where you saw the virtual body

S4* ...you felt that the body in front of you was your body

S5* ...you felt in control of the body that you saw

During the speeded reaction time task, Ifelt the vibrations...

S6* $\quad 0$ (on my real hand)-100 (on the virtual hand)

Please indicate which percentage of the vibrations you felt...

S7* $\quad \ldots$ on your real hand

S8* ... on the virtual hand

Items with * were exploratory. 
up can be ascribed to statistical facilitation. Taken together, the results should indicate whether the illusion differentially affects the RSE and whether the RSE can indeed be used as a measure of the illusion. An advantage of the RSE is that it can be measured during the illusion and that it overcomes the response competition and the exogenous attention issues of the CCE. Hypotheses, sample size and planned analyses were preregistered on the open science framework to create a clear timestamp of the decisions that were made before the experiment was conducted (Note 2).

\section{Methods}

\subsection{Participants}

Twenty-four healthy right-handed participants $\left(M_{\text {age }}=23.0\right.$, range $=20$ 30 , eight males) participated in this experiment. Due to the novelty of the paradigm it was not possible to establish a good estimate of the effect size to determine the sample size. The main effect of trial type has a large effect size in studies that employ a similar task $\left(\eta^{2}: 0.60-0.95\right.$; Bailey et al., 2018, Brandwein et al., 2010, McCracken et al., 2019). However, since there was no study looking into a similar condition effect on the RSE, we could not establish an effect size for the trial type * condition interaction that we were interested in. Miller's race model inequality test (RMI) requires a minimum of 20 trials per trial type, so we followed this recommendation in our task (Kiesel et al., 2007; Ulrich et al., 2007). A recommendation for the minimum number of participants for such a test is not given. As preregistered, a sample of 24 participants was tested because it allowed for the presentation of each counterbalanced order (eight) to an equal number of participants (three). With this sample we had a power of $80 \%$ to detect an effect size $d=0.35$ with our intended mixed-model analysis.

Participants had normal or corrected-to-normal vision (no glasses; contact lenses were allowed), did not suffer from motion sickness and had no history of neurological or psychiatric illness as established by self-report. The research was approved by the local ethics committee and was conducted in accordance with the declaration of Helsinki. All participants provided written informed consent before the start of the experiment and received course credit or gift vouchers for their participation.

\subsection{Head-Mounted Display Setup}

Participants took place in a head-mounted display setup (Lenggenhager et al., 2007; Swinkels et al., 2020a) and were presented with a full-body perspective of their own back. The participants were filmed by a Logitech C920 Pro (Logitech, Lausanne, Switzerland) camera (placed on a tripod) that was positioned 


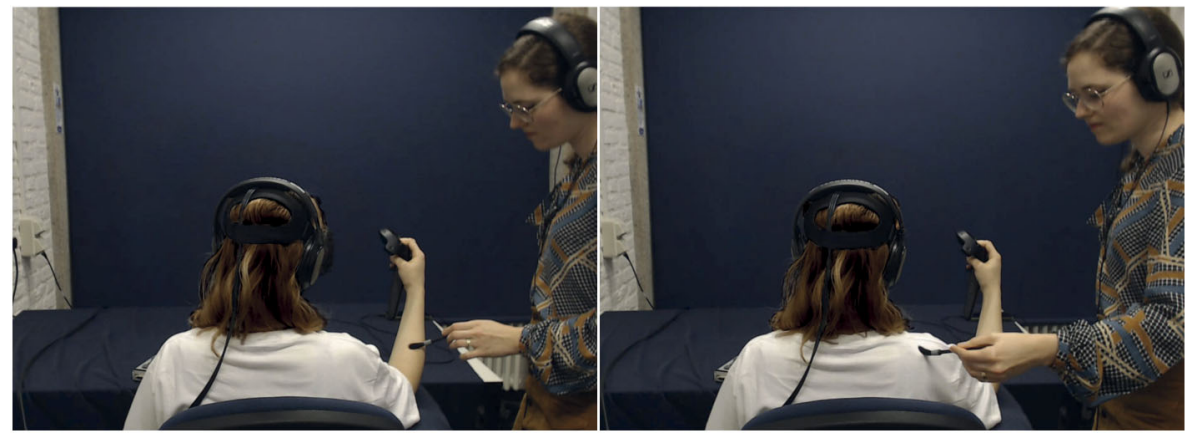

Figure 1. Pictures of the setup depicting a participant holding the Vive controller and the stroking and tapping performed by the experimenter on the underarm (left) and the shoulder (right). The participants sat in an upright position and the experimenter stood next to the participant to administer the stroking and tapping.

$1.5 \mathrm{~m}$ behind them. This camera was connected to an HTC Vive (HTC Corporation, Taoyuan, Taiwan; field of view: 85 degrees, display resolution per eye: $1800 \times 1200$, refresh rate: $90 \mathrm{~Hz}$ ) on which a live video feed or a video of the participant could be presented using a custom-made program that was run on a Dell Precision T3610 computer (Dell, Round Rock, Texas, United States). This program could be used to present a still, a delayed video image, a live feed or a pre-recorded video on the HMD. The participants held a Vive motion tracker in their right hand in order to track the position of their hand during the experiment. They held it in such a way that their thumb and index finger faced the camera positioned behind them (Fig. 1).

\subsection{Experimental Manipulation}

To induce the FBI, participants were stroked and tapped on their underarm and shoulder with a paintbrush in an unpredictable pattern (Fig. 1). The touch that was applied (tapping/stroking) and the location that was touched (underarm/shoulder) changed every $30 \mathrm{~s}$. Each stimulation block lasted two minutes. In the synchronous experimental condition participants were presented with a live video feed of the stimulation on the HMD to create a synchronous experience of the seen and felt tactile stimulation. In the asynchronous control condition participants were presented with a pre-recorded video of the stimulation. This recording was made during the practice block. This enabled us to disrupt the temporal alignment as well as the spatial congruency, which are both considered to be crucial for the induction of body illusions (for a review, see: Kilteni et al., 2015). The temporal alignment was disrupted by presenting participants with the opposite stimulation (i.e., participants felt stroking but saw tapping). Both stroking and tapping were presented at a different pace 
and in their own distinct pattern (slow long brush strokes vs brief spatially constrained taps). Tactile tapping (stroking) by the experimenter was delivered independently from the visual stroking (tapping) in the recorded video and the timing of stimulation in the two modalities thus varied maximally. Spatial congruency was disrupted by simultaneously stimulating different parts of the body, e.g., the shoulder and the underarm. The opposite stimulation (e.g., visual tapping on the shoulder and tactile stroking on the lower arm) furthermore contributed to disrupt spatial multisensory congruency. The experimenter received audio instructions over a set of headphones that indicated which body part had to be stimulated and which stimulation had to be used. Participants listened to white noise over a similar set of headphones to mask the noise of the vibrations.

\subsection{Speeded Detection Task - Stimuli}

\subsubsection{Unisensory Visual Stimulus}

A yellow disc with an apparent diameter of $5 \mathrm{~cm}$ (visual angle: $1.91^{\circ}$ ) was presented on the participant's right hand for $100 \mathrm{~ms}$. The HTC Vive motion tracker was used to determine the position of the participant's hand in space. The tracker was calibrated in such a way that the virtual disc was always presented overlapping the base of the thumb on the back of their hand. Due to the distance between the participant and the camera, the disc was perceived at a viewing distance of approximately $1.5 \mathrm{~m}$. The visual stimulus was only visible on the virtual body through the HMD.

\subsubsection{Unisensory Tactile Stimulus}

The HTC Vive motion tracker was used to present the tactile stimulus. The tactile stimulus was a vibration that was presented for a duration of $100 \mathrm{~ms}$. This vibration was presented in the palm of the hand, on the ball of the thumb to be precise.

\subsubsection{Multisensory Tactile + Visual Stimulus}

The visual and tactile stimuli described above could also be presented simultaneously, whereby the visual stimulus overlapped the body location at the hand where the tactile stimulus was applied.

\subsubsection{Illusion Statements}

To determine illusion strength, participants completed an illusion strength questionnaire. In total, this questionnaire consisted of eight items. The first three items were used to determine illusion strength and were rated on a visual analogue scale ranging from 0 (not at all) to 100 (very strongly). The other questions were included for exploratory purposes and measured ownership, agency and where participants experienced the vibrations that were presented during the redundant signals task (for all items see Table 1). The statements 
were completed after each condition and were presented to participants using Inquisit 4 (Millisecond, 2014).

\subsection{Procedure}

Upon arrival at the lab, the participants received written and verbal instructions about the experiment and provided written informed consent. Next, they were asked to take place in the HMD setup and were shown how to hold the motion tracker. They were instructed to position their right arm on the arm rest in such a way that their shoulder did not overlap with their underarm in the video image. Following these instructions, the participants were fitted with the HMD and a set of headphones that played white noise. Participants were instructed to check whether they were seated in a straight line behind their own body and to check whether the HMD provided a sharp image. The experiment started with a practice block consisting of two stimulation blocks of two minutes.

The participants were asked to pay close attention to the virtual body and the stroking and tapping that was presented to their underarm and shoulder. The practice block served two purposes: to familiarize participants with illusion experiences they may have as a consequence of the synchronized visual and tactile stimulation and to create a video recording that could be used in the control condition. Next, participants completed the speeded detection task. For each condition, the task consisted of five two-minute blocks of stimulation. In the first two-minute block no stimuli were presented yet. This block was used to induce the illusion in the synchronous experimental condition. In the asynchronous control condition participants were also stroked and tapped by the experimenter, but due to the disruption of spatial and temporal congruency no illusion was expected to occur. In the next four blocks the experimenter continued to stroke and tap the participants' underarm and shoulder. Simultaneously, the participant had to respond to the unisensory and multisensory stimuli presented on the virtual and real hand as soon as possible by pressing a button on a custom-made button box with a response accuracy of less than $1 \mathrm{~ms}$ (Technical Support Group, 2019). The response window lasted $1000 \mathrm{~ms}$, regardless of whether participants responded within this window. Each block included nine unisensory visual stimuli, nine unisensory tactile stimuli, nine multisensory visual + tactile stimuli and three catch trials during which no stimuli were presented. Catch trials were included to prevent the build-up of strong expectations towards upcoming stimuli. Interstimulus intervals in all trials were drawn randomly from ranges between 1.5 and $2.5 \mathrm{~s}, 2.5$ and 3.5 $\mathrm{s}$, and 3.5 and $4.5 \mathrm{~s}$. Trials were evenly distributed over the three ranges such that each unisensory trial had three short, three medium and three long interstimulus intervals. The same was done for the other trial types. Stimuli of the four different trial types (tactile, visual, visuotactile, and catch trials) were 
presented randomly and stimuli of the same trial type could be presented maximally twice in a row. In total, participants were presented with 36 unisensory visual trials, 36 unisensory tactile trials, 36 multisensory trials and 12 catch trials per condition. Order of conditions, starting location of the stimulation by the experimenter and stimulation type were counterbalanced [see Supplementary Table S1]. In addition, one half of the participants received the same order of visual stimulation in each corresponding block of the synchronous experimental and the asynchronous control condition (upper half of Supplementary Table S1). The other half received the same order of tactile stimulation (lower half Supplementary Table S1). This resulted in a total of eight counterbalanced orders. After completion of the speeded detection task participants completed the illusion statements. Next, they completed the speeded detection task and the illusion statements for the other condition. At the end of the experiment the participants were interviewed about their illusion experiences. They were asked whether they experienced an illusion, whether they experienced it in both conditions or not and to describe their experiences. In total, the experiment took approximately 60 minutes.

\subsection{Statistical Analyses}

\subsubsection{Illusion Strength}

Assumptions for the corresponding tests were checked. To test illusion strength (H1), separate paired-sample $t$-tests were conducted on the average score of statements 1, 2 and 3 and on these items separately. Exploratory paired-sample $t$-tests were conducted on statements 4-8.

\subsubsection{Speeded Detection Task - Reaction Times}

Trials with RTs $<100 \mathrm{~ms}$ or trials with missing responses were excluded from analysis. Following the preregistered analysis plan we first tried to conduct a linear mixed-effects model analysis using the lmer function of the lme 4 package (version 1.1-21; Bates et al., 2015) in R (R Core Team, 2015) to test whether the reaction times to the multisensory stimuli were faster than the reaction times to the unisensory stimuli. To determine $p$-values we computed Type 3 bootstrapped likelihood ratio tests (using 1000 simulations) as implemented in the mixed function of the package afex (Singmann et al., 2017), which in turn calls the function PBmodcomp of the package pbktest (Halekoh and Højsgaard, 2014). The analysis included a fixed intercept and fixed effects for the factors condition (synchronous, asynchronous), trial type (unisensory visual, unisensory tactile, multisensory) and their interaction, a per-participant random adjustment to the fixed intercept, per-participant random adjustments to the condition, trial type and interaction slopes as well as all possible random correlation terms among the random effects. As preregistered, in case of nonconvergence we first increased the number of iterations and tried different 
optimizers. Next, we tried to simplify the random structure by removing the random correlation terms. In the end none of these efforts resulted in a reliable fit of the model; we therefore resorted to the simpler $2 \times 3$ RM ANOVA that was preregistered as a back-up analysis. The 2 (condition: synchronous, asynchronous) $\times 3$ (trial type: unisensory visual, unisensory tactile, multisensory) RM ANOVA was conducted on the mean RTs per trial type, per condition using the aov function in $\mathrm{R}$ ( $\mathrm{R}$ Core Team, 2015). The same exclusion criteria applied. Tukey-corrected post-hoc tests were conducted to further examine a main effect of trial type and potential interactions with condition.

\subsubsection{Speeded Detection Task - Testing the Race Model}

To assess whether multisensory integration took place, we made use of the RSE by directly comparing the reaction times to the multisensory stimuli (visual + tactile) with the reaction times to the unisensory stimuli (visual or tactile; Colonius and Diederich, 2006; Miller, 1982).

To test whether coactivation took place and the race model was violated, the RMI was conducted separately for both conditions. We made use of the algorithm described by Ulrich et al. (2007). More specifically, we made use of the self-contained Windows program called RMItest that was written for this purpose. This program performs all four steps of the algorithm. It computes: (1) the cumulative density functions (CDF) for every participant and every trial type, i.e., unisensory tactile $\left(G_{\mathrm{x}}\right)$, unisensory visual $\left(G_{\mathrm{y}}\right)$ and multisensory $\left(G_{\mathrm{Z}}\right) ;(2)$ the bound $(B)$ which is the sum of the unisensory CDFs $G_{\mathrm{X}}$ and $G_{\mathrm{y}} ;(3)$ the percentile values for $G_{\mathrm{z}}$ and $B$; and (4) aggregation across participants and the actual test of violation of the race model. For this purpose it makes use of $t$-tests (for more details, see: Ulrich et al., 2007). Percentile values from the 5 th to the 95 th percentile with $5 \%$ increments were used in line with previous studies (Bailey et al., 2018, Brandwein et al., 2010). Since the RMItest program cannot conduct the RMI test when there are too many ties (Note 3) within the RTs, we increased one of the RTs causing a tie by 1 $\mathrm{ms}$ and decreased the other RT by $1 \mathrm{~ms}$ to make the ties unique. This procedure was applied once in the asynchronous condition.

As a secondary analysis of the race model violation we also conducted a permutation test as described by Gondan (2010). This test has a higher power to detect violations of the race model. However, to prevent type I errors due to multiple testing it is necessary to define the set of percentiles for the test $a$ priori. As it was unclear in which percentiles multisensory integration would occur in the current experiment, we followed the suggestion by Kiesel et al. (2007) and conducted the test up to the 25th percentile. Violations of the race model usually occur for the quantiles on the lower end of the reaction times because here it is more likely that integration of the visual and tactile stimulus results in the fulfilment of a response criterion before either of them alone 
satisfies the same criterion (Brandwein et al., 2010; Miller, 1982). In addition, work by Shore et al. (2006) also suggests that multisensory integration occurs early on, before $100 \mathrm{~ms}$. Following the suggestions by Gondan and Minakata (2016), trials with missed responses were not excluded from this analysis but were given an infinite RT instead.

\section{Results}

\subsection{Confirmatory Analyses}

\subsubsection{Illusion Strength}

In line with our first prediction, the paired-sample $t$-test on the average illusion score indicated that participants experienced a stronger illusion in the synchronous experimental condition than in the asynchronous control condition, $t_{23}=-4.55, p<0.001$. The paired-sample $t$-tests on the separate items were in line with our predictions as well. The paired-sample $t$-test on S1 indicated that participants had a stronger experience of feeling the touch of the brush at the location where they saw the virtual body being touched in the synchronous condition than in the asynchronous condition, $t_{23}=-5.13, p<0.001$. For $\mathrm{S} 2$ we found that participants experienced a stronger sensation that the touch that they felt was caused by the brush touching the virtual body in the synchronous condition than in the asynchronous condition, $t_{23}=-2.87, p=0.009$. For S3, finally, participants indicated more strongly that they felt their body to be located where they saw the virtual body in the synchronous condition than in the asynchronous condition, $t_{23}=-2.35, p=0.028$ (see Fig. 2 for averages and individual scores).

\subsubsection{Reaction Times}

The repeated-measures ANOVA with condition and trial type as independent variables on the mean response latencies revealed a significant main effect of trial type. Sphericity was violated so the Greenhouse-Geisser correction was applied, $F_{2,46}=62.58, p<0.001$. We also obtained a significant condition * trial type interaction (sphericity assumed), $F_{2,46}=3.36, p=0.043$. The main effect of condition was not significant, $F_{1,23}=1.02, p=0.323$. In line with our predictions, simple post-hoc comparisons of trial type per condition (Tukey-corrected) revealed that responses to multisensory stimuli were faster than responses to unisensory tactile stimuli, sync: $t_{68.4}=-6.26$, $p<0.001$, async: $\left.t_{68.4}=-8.37, p<0.001\right)$, and unisensory visual stimuli, sync: $t_{68.4}=-9.46, p<0.001$, async: $t_{68.4}=-9.58, p<0.001$. In the synchronous condition, responses to unisensory tactile stimuli were faster than responses to unisensory visual stimuli, $t_{68.4}=-3.19, p=0.006$; no such difference was found for the asynchronous condition, $t_{68.4}=-1.21, p=0.450$. A closer look at Fig. 3 suggests that the interaction effect is driven by a 

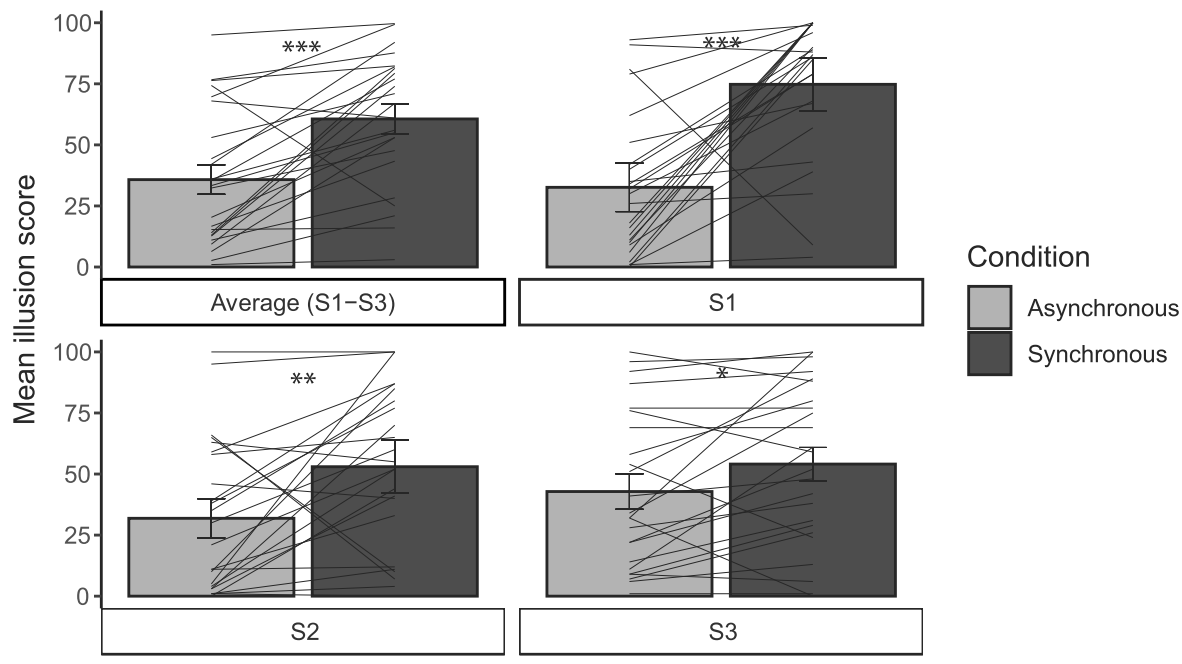

Figure 2. Mean illusion scores displayed separately for each condition per item (S1, S2, S3 and their average). Lines represent data of individual participants for each item in each condition. $* * *, p<0.001$; **, $p<0.01 ; * p<0.05$. The error bars display the $95 \%$ confidence interval.

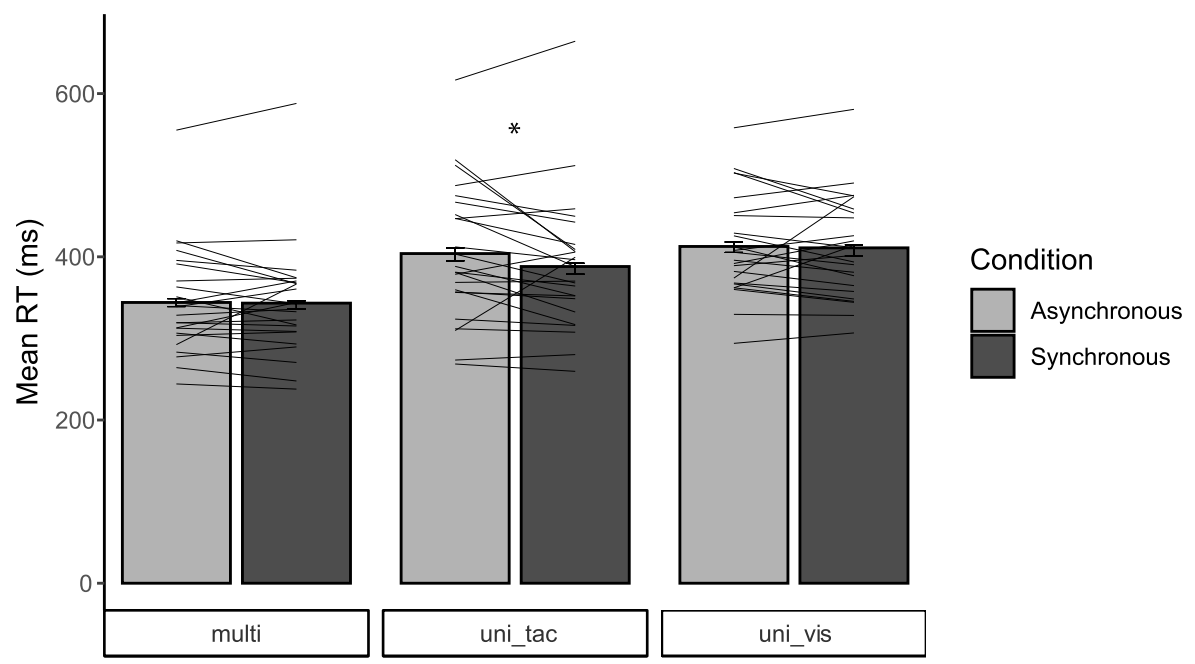

Figure 3. Mean RT (ms) in the multisensory, unisensory tactile and unisensory visual trials displayed per condition. Lines represent individual datapoints for each trial type in each condition. ***, $p<0.001 ; * *, p<0.01 ;^{*} p<0.05$. The error bars display the $95 \%$ confidence interval.

slower response to the unisensory tactile stimuli in the asynchronous condition, and - contrary to our prediction - not by a larger relative gain on the multisensory stimuli in the synchronous condition. This is supported by 
Table 2.

Mean RT and SD of each trial type in each condition. The $p$-values represent the Tukeycorrected post-hoc tests that were conducted following the RM ANOVA

\begin{tabular}{llrlrrr}
\hline & \multicolumn{2}{c}{ Synchronous } & & \multicolumn{2}{c}{ Asynchronous } & \multirow{2}{*}{$p$} \\
\cline { 2 - 3 } & Mean & SD & & Mean & SD & \\
\hline Multi & 341.21 & 93.40 & & 343.96 & 92.88 & 0.910 \\
Uni_tac & 385.28 & 118.20 & & 403.09 & 132.47 & 0.032 \\
Uni_vis & 407.61 & 110.66 & & 411.70 & 103.99 & 0.815 \\
\hline
\end{tabular}

Table 3.

Results of the paired-sample $t$-tests comparing the multisensory CDF with the race model CDF (bound)

\begin{tabular}{lllllr}
\hline Percentile & \multicolumn{2}{c}{ Synchronous condition } & & \multicolumn{2}{c}{ Asynchronous condition } \\
\cline { 2 - 3 } & $t_{23}$ & & & $t_{23}$ & $p$ \\
\hline 0.05 & 4.77 & $<0.001$ & & 3.98 & $<0.001$ \\
0.15 & 6.56 & $<0.001$ & & 6.09 & $<0.001$ \\
0.25 & 5.10 & $<0.001$ & & 6.33 & $<0.001$ \\
0.35 & 3.82 & $<0.001$ & & 5.60 & $<0.001$ \\
0.45 & 3.21 & 0.004 & & 3.45 & 0.002 \\
\hline
\end{tabular}

a post-hoc comparison of condition per trial type, which revealed that participants responded slower to unisensory tactile stimuli in the asynchronous condition compared to the synchronous condition, $t_{41.1}=2.23, p=0.032$. No differences arose for the other trial types (see Table 2 for an overview of the RTs).

\subsubsection{Testing the Race Model}

In the synchronous experimental condition we observed a significant violation of the race model over the first five time points (see Table 3 for full statistical results, see Fig. 4 for a plot of the CDFs belonging to each of the trial types). This result was supported by the permutation test, $t_{23}=8.09, p<0.001$ that was conducted up to the 25 th percentile as preregistered. Contrary to our prediction we also observed a significant violation of the race model over the first five time points in the asynchronous control condition (Note 4). This result was supported by the permutation test as well, $t_{23}=6.24, p<0.001$.

\subsection{Exploratory Analyses}

\subsubsection{Comparing the Race Model Violation Across Conditions}

Contrary to our predictions, a race model violation occurred in both conditions. To directly compare the race model violation in the two conditions we 

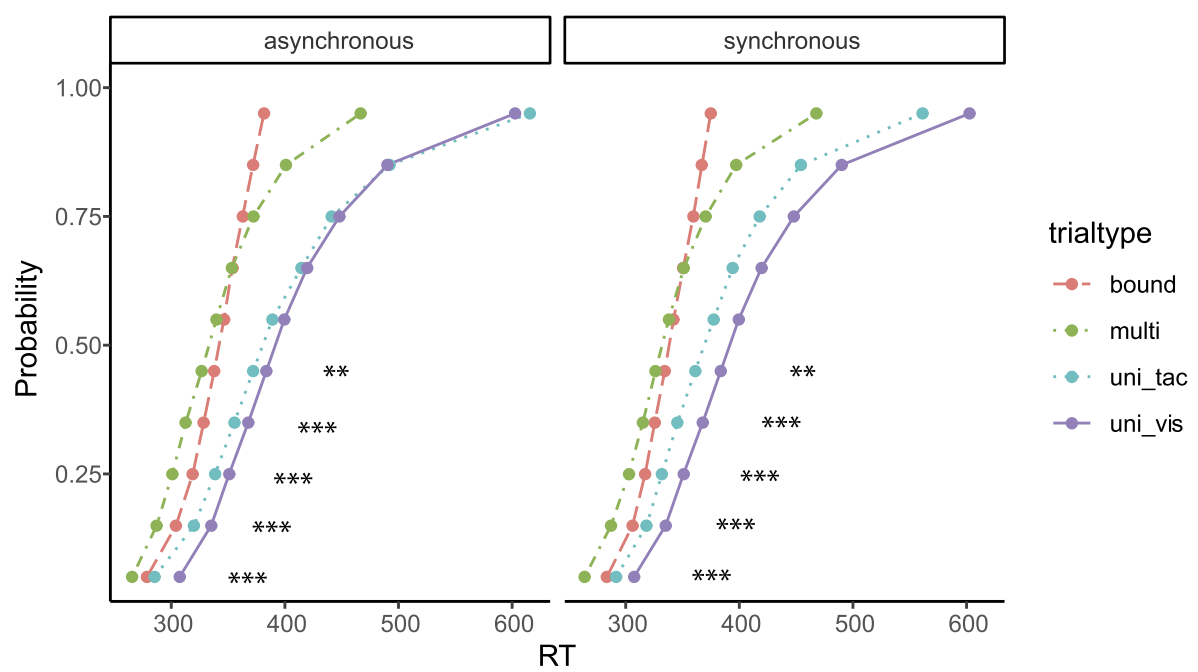

Figure 4. Cumulative distribution functions for each trial type (multi, uni_tac, uni_vis) and the sum of the unisensory stimuli (bound) displayed separately for each condition. ***, $p<0.001$; $* *, p<0.01 ; *, p<0.05$.

exploratively calculated the RMI violation. This was done by subtracting the bound (the summed unisensory CDFs up to a maximum probability of 1) from the multisensory CDF for each condition separately (Fig. 5). For the comparison between the conditions, a single measure of multisensory integration was created by calculating a geometric measure of the RMI violation for each participant. In line with previous studies we calculated the positive area under the RMI violation curve (pAUC) for this purpose (Bailey et al., 2018; Colonius and Diederich, 2006; Nozawa et al., 1994). The pAUC was calculated with the AUC function of the DescTools package in R (Signorell et mult. al., 2020). This function was set up to calculate the trapezoidal area between each time point that produced a RMI violation. The resulting pAUCs for each condition were compared using a paired-samples $t$-test. This $t$-test indicated that condition did not modulate the pAUC, $t_{23}=-0.32, p=0.754\left(M_{\text {sync }}=9.70\right.$, $\mathrm{SD}_{\text {sync }}=7.79, M_{\mathrm{async}}=10.36, \mathrm{SD}_{\text {async }}=8.69$ ). This finding was further supported by a Bayesian paired-samples $t$-test with a default Cauchy prior width of 0.707. The Bayes factor was $\mathrm{BF}_{01}=4.45$, indicating that the observed data are 4.45 times more likely under the null hypothesis that postulates no difference in the positive area under the curve between the two conditions than under the alternative hypothesis that postulates that one condition leads to a larger positive area under the curve than the other. 
condition - asynchronous - - synchronous

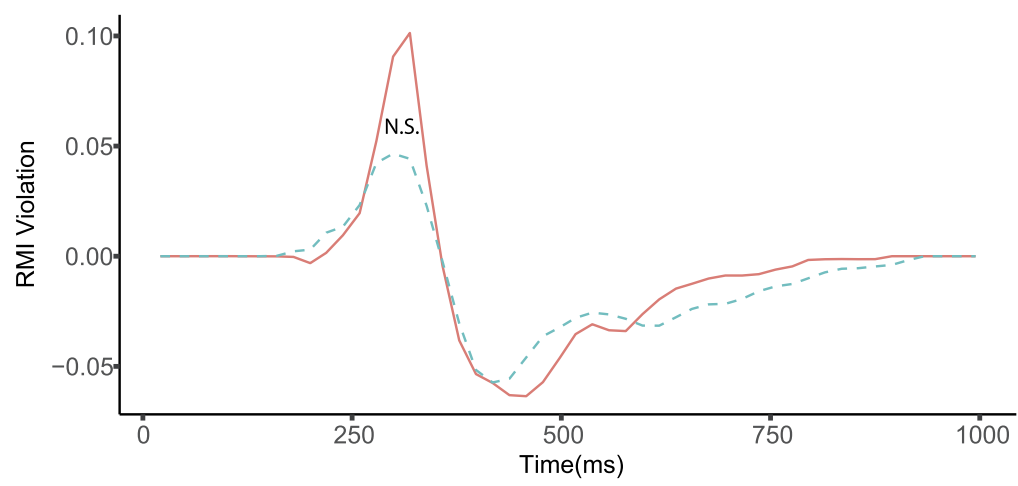

Figure 5. RMI violation plotted by condition. The positive area under the RMI violation curve (pAUC) was not modulated by condition (N.S.).

\subsubsection{Additional Questionnaire Items}

We included a body ownership item in our exploratory illusion strength analyses because this item is often successfully used in other research. In line with our preregistration we indeed found that participants more strongly felt that the virtual body was their body in the synchronous condition $(M=56.13$, $\mathrm{SD}=31.59)$ than in the asynchronous condition $(M=41.00, \mathrm{SD}=30.60)$, $t_{23}=-2.37, p=0.027$. We also explored the sense of agency to investigate if participants would experience a higher sense of agency in the synchronous condition. Participants indeed felt more in control of the virtual body in the synchronous condition $(M=63.17, \mathrm{SD}=31.37)$ compared to the asynchronous condition $(M=34.46, \mathrm{SD}=31.46), t_{23}=-4.42, p<0.001$. Finally, we explored whether the average illusion score based on the first five items differs significantly between the two conditions. We found the illusion in the synchronous condition $(M=60.23, \mathrm{SD}=25.42)$ to be significantly stronger than in the asynchronous condition $(M=36.56, \mathrm{SD}=25.07)$, $t_{23}=-5.13, p<0.001$.

Three final exploratory questions focussed on the subjective experience of transfer of touch to the virtual body, which is considered to be a key component of the FBI (Blanke, 2012; Lenggenhager et al., 2007). In addition to S1 which assessed the transfer of touch from the paintbrush on the participant's body to the virtual body, we were also interested in the transfer of touch from the vibrations that were presented during the redundant signals task on the participant's hand to the virtual hand. On S6, participants indicated that they on average felt the vibrations more on their real hand than on their virtual hand, evidenced by scores below 50 . The paired-samples $t$-test between conditions resulted in a trend towards significance, $t_{23}=1.93, p=0.066$, indicating that 

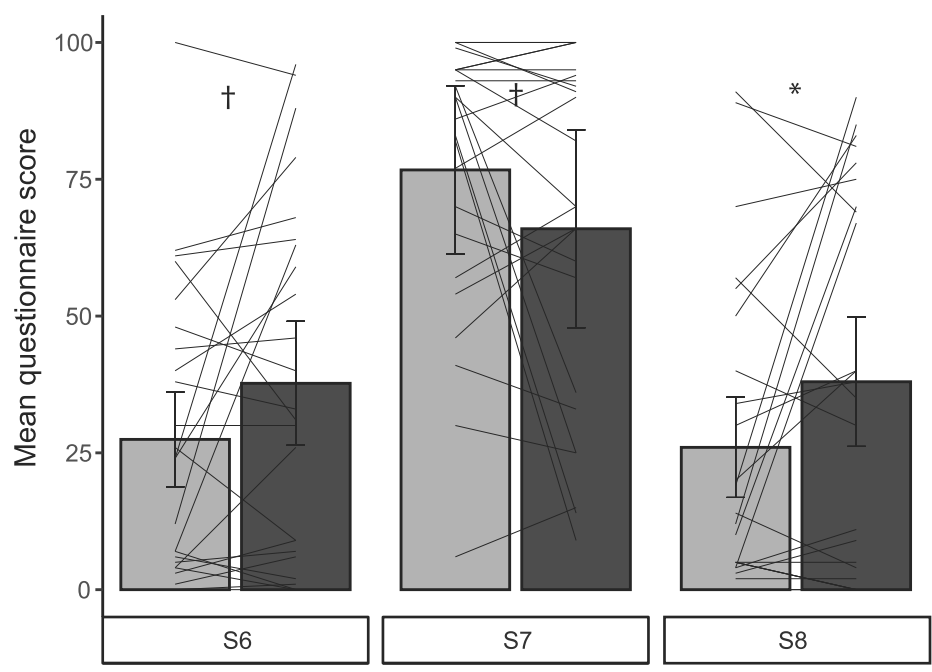

Condition

Figure 6. Mean scores on the exploratory vibration items S6-S8 displayed separately for each condition. Lines represent data points of individual participants for each item in each condition. ${ }^{* *}, p<0.001 ; * *, p<0.01 ; *, p<0.05 ; \dagger, p<0.1$. The error bars display the $95 \%$ confidence interval.

participants on average felt the vibrations more on their virtual hand in the synchronous condition $(M=37.71, \mathrm{SD}=32.70)$ than in the asynchronous condition $(M=27.46, \mathrm{SD}=26.57)$. The analyses of $\mathrm{S} 7$ and $\mathrm{S} 8$ showed a similar pattern with participants indicating a lower percentage of felt vibrations on their real hand in the synchronous condition $(M=65.96, \mathrm{SD}=31.74)$ than in the asynchronous condition $(M=76.71, \mathrm{SD}=25.50)$ on $\mathrm{S} 7, t_{23}=-1.95$, $p=0.063$, and a higher percentage of felt vibrations on the virtual hand in the synchronous condition $(M=38.00, \mathrm{SD}=34.26)$ than in the asynchronous condition $(M=26.00, \mathrm{SD}=28.48)$ on $\mathrm{S} 8, t_{23}=2.10, p=0.047$. Note that participants still felt the majority of vibrations on their real hand (evidenced by the low scores on S6 and the high scores on S7; Fig. 6), but that at least a reasonable percentage of vibrations seemed to transfer towards the virtual hand. This percentage appears to be larger for the synchronous condition in line with the expected transfer of touch in this condition (Fig. 6, S8).

\section{Discussion}

In the present study we investigated whether the RSE can be used as an objective measure of the FBI by directly tapping into the multisensory integration underlying the illusion. The RSE was chosen as an alternative to the CCE task because the CCE task has led to conflicting results in the literature and 
may be explained by response conflict and exogenous cueing rather than multisensory integration (Forster et al., 2008, Marini et al., 2017; Patané et al., 2019; Spence et al., 2004). To test the RSE as an alternative objective measure, we investigated whether the RSE would be differentially affected by our synchronous experimental condition in which an illusion was induced and our asynchronous control condition in which no illusion was induced. We predicted that participants would respond faster to the multisensory stimuli than to the unisensory stimuli in both conditions, but that this relative speeding up would only be accompanied by a violation of the race model in the synchronous condition, reflective of an integration of the visual and vibrotactile stimulus in line with the CCE literature (Maselli and Slater, 2014).

Although we found a significantly stronger illusion in the synchronous condition as compared to the asynchronous control condition and significantly faster responses to the multisensory stimuli than to the unisensory stimuli in both conditions, our hypotheses regarding the race model violation were not confirmed. Instead, we found a violation of the race model in both conditions, indicating that integration of the stimuli took place in both the synchronous and the asynchronous condition, regardless of whether an illusion was induced. Our exploratory analysis of the positive area under the curve suggests that the magnitude of the violation did not differ between the conditions. Interestingly, we did find a significant difference between the two conditions in the RTs to the unisensory tactile stimuli. Taken together, these results suggest that the RSE is not suitable as an objective measure of the FBI in its original form because it cannot distinguish between a condition in which an illusion was induced and a condition in which no illusion was induced. However, the difference in RT obtained between the conditions on the unisensory tactile stimuli suggests that the RSE task may be used as an objective measure to determine whether an illusion occurred.

The fact that we found a violation of the race model in both conditions and not just in our synchronous illusion condition may be explained in at least three ways. In an experiment where multisensory integration in older and younger adults was compared, Couth et al. (2018) demonstrated that the magnitude of race model violation in a group or condition may increase when the responses to unisensory stimuli slow down. In the current study participants responded more slowly to the unisensory tactile stimuli in the asynchronous condition than to the same tactile stimuli in the synchronous condition. This could explain why we found a race model violation in the asynchronous condition. We conducted the analyses suggested by Couth et al. (2008) to test whether this explanation might hold for our data (see supplementary material for analyses). However, unlike Couth et al. (2018), we did not find a significant difference between the two conditions when the fastest unisensory responses were compared. Nor did we find a significant correlation between 
the fastest unisensory response and the amount of race model violation in the asynchronous condition. This suggests that the slower responses to unisensory stimuli in the asynchronous condition (driven by slow responses to the tactile stimuli in our experiment) do not provide a likely explanation for the unexpected race model violation in that condition.

A second possibility is that the lack of a significant difference in the RSE between conditions can be attributed to the paradigm that was used. The FBI with a third-person perspective (3pp) on the body usually results in a somewhat weaker illusion of ownership when compared to the full body ownership illusion (FBOI) that employs a first-person perspective (1pp; e.g., Galvan Debarba et al., 2017; Gorisse et al., 2017). Still, the current paradigm and study design were effective in generating consistent differences between conditions on the illusion questionnaire, confirming previous research on the FBI (e.g., Aspell et al., 2009; Pamment and Aspell, 2017; Pomés and Slater, 2013; Salomon et al., 2013; Swinkels et al., 2020a, b). Furthermore, and most importantly in light of the study's aim to investigate the RSE as an objective measure of the FBI, analysis of individual items indicated that participants experienced a clear transfer of touch to the virtual body in the synchronous condition, as compared to the asynchronous condition. That is, following previous research on the CCE (Maselli and Slater, 2014), we had hypothesized that the transfer of touch to the virtual body should increase multisensory integration of bimodal stimuli in the synchronous condition, whereas the absence of transfer of touch to the virtual body in the asynchronous condition should obstruct multisensory integration. Considering that the RSE task does not show any difference between conditions despite the clear difference in the reported transfer of touch suggests that the RSE is simply unsuitable as a reliable measure of the FBI.

A third, more likely explanation for the absence of a difference in the RSE between conditions, is that the sheer simultaneous occurrence of the visual and vibrotactile stimulus was enough to result in multisensory integration, regardless of the fact that the stimuli were presented on two different bodies and the fact that these bodies were not integrated in absence of the illusion. The three general principles of multisensory integration dictate that integration should be more likely when the unisensory stimuli (1) arise from approximately the same location; (2) occur at approximately the same time; and (3) evoke relatively weak responses when they are presented separately (Holmes and Spence, 2005; Spence, 2013).

Although the spatial rule does not seem to apply here, as the virtual body and the real body were perceived as separate in the majority of the cases in the asynchronous condition, the multisensory integration temporal rule may well have been sufficient to support (transient) multisensory integration between the visual disc on the virtual hand and the synchronous tactile stimulus on the 
participant's real hand. We therefore think that multisensory integration occurs automatically following low-level synchronicity, irrespective of whether multisensory stimuli are functionally linked at a higher level (e.g., being considered as part of one body). The experience of a unified body representation should therefore be considered as a consequence of the multisensory integration of the visual and tactile stimulation presented to the virtual and the real body and not as a prerequisite of multisensory integration (as is often suggested in the literature on the CCE).

In addition to the unexpected finding that multisensory integration took place in the asynchronous condition, Bayesian analysis indicated that multisensory integration was equally strong in both conditions. This suggests that the automatic integration of multisensory (disc and vibrator) stimuli presented to the hand, took place in parallel and independent from the (illusory or real) body representations that were activated in parallel. The latter finding is consistent with a representational model of body construction in which separate channels of multisensory information (i.e., the tapping and stroking by an experimenter; the occasional presence of vibrating discs on the hand) are processed in parallel, whereby the body representation that is based on evidence in one channel does not necessarily affect the processing of stimuli in a separate parallel channel. We suggest that only when stimuli in parallel channels are repeatedly and reliably found to be simultaneous or related in time, the brain may link these channels into a joint multisensory representation. In the case of the present experiment, the illusory body representation that was evoked through the synchronous visuotactile stimulation by the experimenter, was not integrated with the more variable visual and tactile stimulation on the hand. Perhaps, if vibrotactile stimuli on the hand had been used to induce the illusion, the RSE task would have been able to distinguish between multisensory integration in both conditions. This, however, would strongly reduce the application of the RSE task as it would mean that the same stimuli that are used to measure the presence or absence of an illusion, need to be used to induce the illusion in the first place.

Interestingly, whereas no differences were observed in the processing of multisensory stimuli in the synchronous and asynchronous conditions, reaction times to unisensory tactile stimuli were found to be slower in the asynchronous stimulation. We believe that this relative slowing down of responses to unisensory tactile stimuli in the asynchronous condition may be attributed to attention having to switch from the virtual body to the real body in the asynchronous condition. More precisely, as participants were instructed to pay attention to the visual stroking and tapping during the RSE task, and considering that visual stimuli are usually dominant over somatosensory stimuli (Rock and Victor, 1964), we assume that participants' attention in both conditions was primarily directed at the virtual body. Considering that our exploratory 
analyses indicated that tactile stimuli were felt to originate more often on the virtual hand in the synchronous condition than in the asynchronous condition (see Fig. 6, S6 and S8), this implies that tactile stimuli in the synchronous condition were presented more frequently inside the spotlight of attention than tactile stimuli in the asynchronous condition. Previous literature has securely established that the stimuli in the spotlight of attention are facilitated and responded to faster than stimuli presented outside the focus of attention (Kennett et al., 2001; Posner et al., 1980; Tipper et al., 2001).

In further support of our reasoning that tactile stimuli in the asynchronous condition were presented outside the focus of attention more often and required a shift of attention from the virtual body to the real body, we conducted an analysis of the variance in reaction times for both the unisensory tactile and the unisensory visual stimuli (see supplementary material). We hypothesized that if attention is primarily focussed on the virtual body (visual) we would see a higher variance for the reaction times for unisensory tactile stimuli in the asynchronous condition. We indeed obtained a trend towards higher RT variance in the asynchronous condition than in the synchronous condition for unisensory tactile stimuli but not for unisensory visual stimuli. These findings nicely support our interpretation that tactile stimuli in the asynchronous condition required more frequent shifts of attention to the location of the real body, whereas with visual stimuli no such shift was necessary as these stimuli were already presented in the spotlight of attention. Considering that the slower responses to tactile stimuli in the asynchronous condition reflect an unexpected finding, future research is necessary to replicate these findings in a confirmatory analysis. Potentially, the finding in tactile reaction times could provide a starting point for a new objective measure to distinguish between conditions with and without illusory body embodiment.

Our exploratory questions on the experienced transfer of touch of the vibrations to the virtual body carefully suggest that participants indeed experienced a larger percentage of the vibrations on the virtual body in the synchronous compared to the asynchronous condition, supporting the notion that participants may switch less in the synchronous condition. However, the percentages also indicate that the majority of the vibrations was still felt on the real body and not on the virtual body. The fact that participants do not experience the vibrations on the virtual body all the time in the synchronous condition may indicate that the illusion is not present continuously but may actually break and re-emerge during the tactile stimulation with the paintbrush. Kokkinara and Slater (2014) measure the illusion through time and record breaks in the illusion by asking participants to indicate transitions from experiencing the illusion to not experiencing the illusion. Additional questions were used to find out why few breaks were reported (if this was the case) and to find out what the reasons were for transitions between illusion and no illusion. Our 
findings on the exploratory transfer of touch questions suggest that it may be worthwhile to combine the breaks approach by Kokkinara and Slater (2014) with the traditional method of measuring illusion strength. More and more research seems to point in the direction that the illusion should be considered as an all-or-nothing phenomenon where the strength of the illusion may be less indicative of whether an illusion occurred (Kalckert and Ehrsson, 2014; Swinkels et al., 2020b). Instead it may be more important to ask if an illusion occurred, when it occurred and for how long.

\subsection{Limitations and Directions for Future Research}

As discussed in the Introduction, a problem that often occurs with methods that are intended to measure body illusions is that there is often a dissociation between the questionnaires that measure the subjective experience of the illusion and other measures like proprioceptive drift, SCR and HRD that are supposed to measure the illusion in an objective way (Ma and Hommel, 2013; Maselli and Slater, 2013; Metral et al., 2017; Riemer et al., 2015, 2019). Our unisensory tactile effect was no exception, as we did not find a correlation between various indices of the illusion and the reaction times to the unisensory tactile stimuli (see Supplementary Table S2). An interesting point in this regard was made by Rohde et al. (2011) who stated that "the more plausibly it can be argued that the same mechanism brings about both the feeling of ownership and the behavioural correlate in question, the stronger one can be seen as an indicator of the other". This suggests that, in most cases, the subjective measures of the illusion and the objective measures measure different aspects of the illusion. They should therefore not be used instead of each other but rather in a complementary fashion where they each can provide complementary information on whether an illusion was experienced. In addition, the field may benefit from adopting open science practices (Munafò et al., 2017). Increasing the transparency in reporting may aid in reaching standards for testing the occurrence and strength of the illusion, preparing the data for analysis and could ultimately help in reducing the number of conflicting results and a file drawer problem that may in part contribute to the conflicts in the literature.

Based on the present study, we had to conclude that the RSE task is not suitable as a measure of the illusion because it does not discriminate between the synchronous condition in which an illusion was induced and the asynchronous condition in which no illusion was induced. However, the significant difference between the two conditions on the unisensory tactile stimulus may provide an interesting starting point for future research. This effect may directly tap into the transfer of touch by showing that participants actually need to switch less often between the virtual and the real body when the illusion is induced. Future research might consider removing the multisensory trials and increasing the number of unisensory visual and tactile trials to see if this effect 
holds. Keeping the unisensory visual trials is vital as this ensures that participants actually need to perform the switching between the virtual and the real body in the asynchronous condition.

A comparable task may be designed for the RHI and FBOI. Although the RHI uses a first-person perspective on the rubber hand, the rubber hand and the participant's real hand usually do not overlap. When asynchronous stimulation is applied in this paradigm, the rubber hand and the participant's real hand are experienced as two separate hands (Kalckert and Ehrsson, 2012, 2014; Samad et al., 2015). A similar switching cost may therefore be expected in the asynchronous condition for this paradigm. Two competing predictions can be made for the FBOI. In the FBOI there is full spatial overlap of the virtual and the real body. If attention to the visual and tactile stimuli is considered from a spatial reference frame it may be hypothesized that no switching cost will be incurred for the tactile stimuli because the visual and tactile stimuli are presented in the same spatial location (Posner et al., 1980). However, if attention to the stimuli is considered from an object-based perspective (Chen, 2012), a switching cost may still be incurred. The virtual/fake body and the real body are usually experienced as separate entities following asynchronous stimulation (e.g., Petkova and Ehrsson, 2008; Slater et al., 2010). If participants attend the virtual body as instructed, they have to switch to the real body when a tactile stimulus is presented.

\subsection{Conclusion}

In the current study we ruled out the RSE task as a potential technique to objectively determine the presence of bodily illusions and found that the synchronous presentation of visual and tactile bodily stimuli provides a powerful trigger for multisensory integration, irrespective of whether participants experience a body illusion or not. This finding argues against the assumption in the CCE literature that the induction of a bodily illusion will cause multimodal stimuli to become integrated, whereas no such integration takes place when no body illusion is induced. Interestingly, our analyses of the unisensory tactile reaction times point to a potential alternative measure that may be used to objectively distinguish whether a body illusion is present or not. Future research is necessary to replicate and further investigate extending this measure to other types of body illusions. We hope our research efforts will contribute to the development of new objective and useful measures and a deeper understanding of the mechanisms that underlie our sense of embodiment and its transfer to virtual bodies. 


\section{Acknowledgements}

We would like to thank Erik van den Berge for his technical support. We would like to thank Rob Bulterman and Peiying Zuo for their willingness to participate in a pilot of the experimental setup.

\section{Funding}

This work was supported by the Behavioural Science Institute, Radboud University, Nijmegen, The Netherlands.

\section{Supplementary Material}

Supplementary material is available online at: https://doi.org/10.6084/m9.figshare.14307908

\section{Notes}

1. In the present study, illusion questions were used in a within-subject context and complemented with interviews to reduce the amount of ambiguity to a minimum.

2. https://osf.io/mu2a4/?view_only=f1ace8f4393646288db8cd76121b4b6f https://osf.io/vbyr7?view_only=040e856952364fcca48c04e48c1988c0

3. Ties occur when a RT occurs more than once in a dataset, i.e., not all RT values are unique.

4. As can be seen in Figure 3, some participants also experienced some sort of illusion in the asynchronous control condition. Removal of these participants from the race model analysis does not change the results. The race model is still violated in both the synchronous and the asynchronous condition. However, instead of violation up to the 45 th percentile, the race model is violated up to the 35 th percentile.

\section{References}

Abdulkarim, Z. and Ehrsson, H. H. (2016). No causal link between changes in hand position sense and feeling of limb ownership in the rubber hand illusion, Atten. Percept. Psychophys. 78, 707-720. DOI:10.3758/s13414-015-1016-0.

Aimola Davies, A. M., White, R. C. and Davies, M. (2013). Spatial limits on the nonvisual self-touch illusion and the visual rubber hand illusion: subjective experience of the illusion and proprioceptive drift, Consc. Cogn. 22, 613-636. DOI:0.1016/j.concog.2013.03.006.

Altschuler, E. L. and Ramachandran, V. S. (2007). A simple method to stand outside oneself, Perception 36, 632-634. DOI:10.1068/p5730. 
Armel, K. C. and Ramachandran, V. S. (2003). Projecting sensations to external objects: evidence from skin conductance response, Proc. R. Soc. B Biol. Sci. 270, 1499-1506. DOI:10. 1098/rspb.2003.2364.

Aspell, J. E., Lenggenhager, B. and Blanke, O. (2009). Keeping in touch with one's self: multisensory mechanisms of self-consciousness, PLOS ONE 4, e6488. DOI:10.1371/journal.pone. 0006488.

Bailey, H. D., Mullaney, A. B., Gibney, K. D. and Kwakye, L. D. (2018). Audiovisual integration varies with target and environment richness in immersive virtual reality, Multisens. Res. 31, 689-713. DOI:10.1163/22134808-20181301.

Bates, D., Mächler, M., Bolker, B. and Walker, S. (2015). Fitting linear mixed-effects models using lme4, J. Stat. Softw. 67. DOI:10.18637/jss.v067.i01.

Blanke, O. (2012). Multisensory brain mechanisms of bodily self-consciousness, Nat. Rev. Neurosci. 13, 556-571. DOI:10.1038/nrn3292.

Botvinick, M. and Cohen, J. (1998). Rubber hands 'feel' touch that eyes see, Nature 391, 756. DOI: $10.1038 / 35784$.

Brandwein, A. B., Foxe, J. J., Russo, N. N., Altschuler, T. S., Gomes, H. and Molholm, S. (2010). The development of audiovisual multisensory integration across childhood and early adolescence: a high-density electrical mapping study, Cereb. Cortex 21, 1042-1055. DOI:10. 1093/cercor/bhq170.

Chen, Z. (2012). Object-based attention: a tutorial review, Atten. Percept. Psychophys. 74, 784802. DOI:10.3758/s13414-012-0322-z.

Colonius, H. and Diederich, A. (2006). The race model inequality: interpreting a geometric measure of the amount of violation, Psychol. Rev. 113, 148-154. DOI:10.1037/0033-295x. 113.1.148.

Couth, S., Gowen, E. and Poliakoff, E. (2018). Using race model violation to explore multisensory responses in older adults: enhanced multisensory integration or slower unisensory processing? Multisens. Res. 31, 151-174. DOI:10.1163/22134808-00002588.

Ehrsson, H. (2012). The concept of body ownership and its relation to multisensory integration, in: The New Handbook of Multisensory Processes, B. E. Stein (Ed.), pp. 775-792. Cambridge, MA, USA. [Reprinted from Ehrsson, H. H. (2007). The experimental induction of out-of-body experiences, Science 317, 1048.]

Ehrsson, H. H., Holmes, N. P. and Passingham, R. E. (2005). Touching a rubber hand: feeling of body ownership is associated with activity in multisensory brain areas, J. Neurosci. 25, 10564-10573. DOI:10.1523/jneurosci.0800-05.2005.

Ferri, F., Chiarelli, A. M., Merla, A., Gallese, V. and Costantini, M. (2013). The body beyond the body: expectation of a sensory event is enough to induce ownership over a fake hand, Proc. R. Soc. B Biol. Sci. 280, 20131140. DOI:10.1098/rspb.2013.1140.

Forster, B., Forster, B. and Pavone, E. F. (2008). Electrophysiological correlates of crossmodal visual distractor congruency effects: evidence for response conflict, Cogn. Affect. Behav. Neurosci. 8, 65-73. DOI:10.3758/CABN.8.1.65.

Gallagher, S. (2006). How the Body Shapes the Mind. Clarendon Press, Oxford, UK.

Galvan Debarba, H., Bovet, S., Salomon, R., Blanke, O., Herbelin, B. and Boulic, R. (2017). Characterizing first and third person viewpoints and their alternation for embodied interaction in virtual reality, PLOS ONE 12, e0190109. DOI:10.1371/journal.pone.0190109. 
Gentile, G., Guterstam, A., Brozzoli, C. and Ehrsson, H. H. (2013). Disintegration of multisensory signals from the real hand reduces default limb self-attribution: an fMRI study, J. Neurosci. 33, 13350-13366. DOI:10.1523/jneurosci.1363-13.2013.

Gondan, M. (2010). A permutation test for the race model inequality, Behav. Res. Meth. 42, 23-28. DOI:10.3758/brm.42.1.23.

Gondan, M. and Minakata, K. (2016). A tutorial on testing the race model inequality, Atten. Percept. Psychophys. 78, 723-735. DOI:10.3758/s13414-015-1018-y.

Gorisse, G., Christmann, O., Amato, E. A. and Richir, S. (2017). First- and third-person perspectives in immersive virtual environments: presence and performance analysis of embodied users, Front. Robotics AI 4, 33. DOI:10.3389/frobt.2017.00033.

Guterstam, A., Gentile, G. and Ehrsson, H. H. (2013). The invisible hand illusion: multisensory integration leads to the embodiment of a discrete volume of empty space, J. Cogn. Neurosci. 25, 1078-1099. DOI:10.1162/jocn_a_00393.

Guterstam, A., Björnsdotter, M., Gentile, G. and Ehrsson, H. H. (2015). Posterior cingulate cortex integrates the senses of self-location and body ownership, Curr. Biol. 25, 1416-1425. DOI:10.1016/j.cub.2015.03.059.

Haggard, P. and Wolpert, D. M. (2005). Disorders of body scheme, in: Higher-Order Motor Disorders, H.-J. Freund, M. Jeannerod, M. Hallett and R. C. Leiguarda (Eds), pp. 261-272. Oxford University Press, Oxford, UK.

Halekoh, U. and Højsgaard, S. (2014). A Kenward-Roger approximation and parametric bootstrap methods for tests in linear mixed models — the R package pbkrtest, J. Stat. Softw. 59. DOI:10.18637/jss.v059.i09.

Hara, M., Salomon, R., van der Zwaag, W., Kober, T., Rognini, G., Nabae, H., Yamamoto, A., Blanke, O. and Higuchi, T. (2014). A novel manipulation method of human body ownership using an fMRI-compatible master-slave system, J. Neurosci. Methods 235, 25-34. DOI:10. 1016/j.jneumeth.2014.05.038.

Holle, H., McLatchie, N., Maurer, S. and Ward, J. (2011). Proprioceptive drift without illusions of ownership for rotated hands in the "rubber hand illusion" paradigm, Cogn. Neurosci. 2, 171-178. DOI:10.1080/17588928.2011.603828.

Holmes, N. P. and Spence, C. (2005). Multisensory integration: space, time and superadditivity, Curr. Biol. 15, R762-R764. DOI:10.1016/j.cub.2005.08.058.

Kalckert, A. and Ehrsson, H. H. (2012). Moving a rubber hand that feels like your own: a dissociation of ownership and agency, Front. Hum. Neurosci. 6, 40. DOI:10.3389/fnhum. 2012.00040.

Kalckert, A. and Ehrsson, H. H. (2014). The moving rubber hand illusion revisited: comparing movements and visuotactile stimulation to induce illusory ownership, Consc. Cogn. 26, 117 132. DOI:10.1016/j.concog.2014.02.003.

Kennett, S., Eimer, M., Spence, C. and Driver, J. (2001). Tactile-visual links in exogenous spatial attention under different postures: convergent evidence from psychophysics and ERPs, J. Cogn. Neurosci. 13, 462-478. DOI:10.1162/08989290152001899.

Kiesel, A., Miller, J. and Ulrich, R. (2007). Systematic biases and Type I error accumulation in tests of the race model inequality, Behav. Res. Methods 39, 539-551. DOI:10.3758/ bf03193024. 
Kilteni, K., Maselli, A., Kording, K. P. and Slater, M. (2015). Over my fake body: body ownership illusions for studying the multisensory basis of own-body perception, Front. Hum. Neurosci. 9, 141. DOI:10.3389/fnhum.2015.00141.

Kokkinara, E. and Slater, M. (2014). Measuring the effects through time of the influence of visuomotor and visuotactile synchronous stimulation on a virtual body ownership illusion, Perception 43, 43-58. DOI:10.1068/p7545.

Lane, T., Yeh, S.-L., Tseng, P. and Chang, A.-Y. (2017). Timing disownership experiences in the rubber hand illusion, Cogn. Res. Princ. Implic. 2, 4. DOI:10.1186/s41235-016-0041-4.

Lenggenhager, B., Tadi, T., Metzinger, T. and Blanke, O. (2007). Video ergo sum: manipulating bodily self-consciousness, Science 317, 1096-1099. DOI:10.1126/science.1143439.

Longo, M. R., Schüür, F., Kammers, M. P. M., Tsakiris, M. and Haggard, P. (2008). What is embodiment? A psychometric approach, Cognition 107, 978-998. DOI:10.1016/j.cognition. 2007.12.004.

Ma, K. and Hommel, B. (2013). The virtual-hand illusion: effects of impact and threat on perceived ownership and affective resonance, Front. Psychol. 4, 604. DOI:10.3389/fpsyg.2013. 00604.

Marini, F., Romano, D. and Maravita, A. (2017). The contribution of response conflict, multisensory integration, and body-mediated attention to the crossmodal congruency effect, Exp. Brain Res. 235, 873-887. DOI:10.1007/s00221-016-4849-4.

Maselli, A. and Slater, M. (2013). The building blocks of the full body ownership illusion, Front. Hum. Neurosci. 7, 83. DOI:10.3389/fnhum.2013.00083.

Maselli, A. and Slater, M. (2014). Sliding perspectives: dissociating ownership from selflocation during full body illusions in virtual reality, Front. Hum. Neurosci. 8, 693. DOI:10. 3389/fnhum.2014.00693.

McCracken, H. S., Murphy, B. A., Glazebrook, C. M., Burkitt, J. J., Karellas, A. M. and Yielder, P. C. (2019). Audiovisual multisensory integration and evoked potentials in young adults with and without attention-deficit/hyperactivity disorder, Front. Hum. Neurosci. 13, 95. DOI:10.3389/fnhum.2019.00095.

Metral, M., Gonthier, C., Luyat, M. and Guerraz, M. (2017). Body schema illusions: a study of the link between the rubber hand and kinesthetic mirror illusions through individual differences, BioMed Res. Int. 2017, 6937328. DOI:10.1155/2017/6937328.

Miller, J. (1982). Divided attention: evidence for coactivation with redundant signals, Cogn. Psychol. 14, 247-279. DOI:10.1016/0010-0285(82)90010-X.

Miller, J. (1986). Timecourse of coactivation in bimodal divided attention, Percept. Psychophys. 40, 331-343. DOI:10.3758/BF03203025.

Millisecond (2014). Inquisit 4, version 4.0.8.0. Retrieved from https://www.millisecond.com.

Minakata, K. and Gondan, M. (2018). Differential coactivation in a redundant signals task with weak and strong go/no-go stimuli, Q. J. Exp. Psychol. 72, 922-929. DOI:10.1177/ 1747021818772033.

Munafò, M. R., Nosek, B. A., Bishop, D. V. M., Button, K. S., Chambers, C. D., Percie du Sert, N., Simonsohn, U., Wagenmakers, E.-J., Ware, J. J. and Ioannidis, J. P. A. (2017). A manifesto for reproducible science, Nat. Hum. Behav. 1, 0021. DOI:10.1038/s41562-016-0021.

Nozawa, G., Reuter-Lorenz, P. A. and Hughes, H. C. (1994). Parallel and serial processes in the human oculomotor system: bimodal integration and express saccades, Biol. Cybern. 72, 19-34. DOI:10.1007/BF00206235. 
Pamment, J. and Aspell, J. E. (2017). Putting pain out of mind with an 'out of body' illusion, Eur. J. Pain 21, 334-342. DOI:10.1002/ejp.927.

Patané, I., Cardinali, L., Salemme, R., Pavani, F., Farnè, A. and Brozzoli, C. (2019). Action planning modulates peripersonal space, J. Cogn. Neurosci. 31, 1141-1154. DOI:10.1162/ jocn_a_01349.

Pavani, F., Spence, C. and Driver, J. (2000). Visual capture of touch: out-of-the-body experiences with rubber gloves, Psychol. Sci. 11, 353-359. DOI:10.1111/1467-9280.00270.

Petkova, V. I. and Ehrsson, H. H. (2008). If I were you: perceptual illusion of body swapping, PLoS ONE 3, e3832. DOI:10.1371/journal.pone.0003832.

Petkova, V. I. and Ehrsson, H. H. (2009). When right feels left: referral of touch and ownership between the hands, PLOS ONE 4, e6933. DOI:10.1371/journal.pone.0006933.

Petkova, V. I., Khoshnevis, M. and Ehrsson, H. H. (2011). The perspective matters! Multisensory integration in ego-centric reference frames determines full-body ownership, Front. Psychol. 2, 35. DOI:10.3389/fpsyg.2011.00035.

Pomés, A. and Slater, M. (2013). Drift and ownership toward a distant virtual body, Front. Hum. Neurosci. 7, 908. DOI:10.3389/fnhum.2013.00908.

Posner, M. I., Snyder, C. R. and Davidson, B. J. (1980). Attention and the detection of signals, J. Exp. Psychol. Gen. 109, 160-174. DOI:10.1037/0096-3445.109.2.160.

Preston, C., Kuper-Smith, B. J. and Ehrsson, H. H. (2015). Owning the body in the mirror: the effect of visual perspective and mirror view on the full-body illusion, Sci. Rep. 5, 18345. DOI:10.1038/srep18345.

Preuss, N. and Ehrsson, H. H. (2019). Full-body ownership illusion elicited by visuovestibular integration, J. Exp. Psychol. Hum. Percept. Perform. 45, 209-223. DOI:10.1037/ xhp0000597.

R Core Team (2015). R: a Language and Environment for Statistical Computing. R Foundation for Statistical Computing, Vienna, Austria. https://cran.r-project.org.

Raab, D. H. (1962). Statistical facilitation of simple reaction times, Trans. N. Y. Acad. Sci. U. S. A. 24, 574-590. DOI:10.1111/j.2164-0947.1962.tb01433.x.

Raz, G., Gurevitch, G., Vaknin, T., Aazamy, A., Gefen, I., Grunstein, S., Azouri, G. and Goldway, N. (2020). Electroencephalographic evidence for the involvement of mirror-neuron and error-monitoring related processes in virtual body ownership, NeuroImage 207, 116351. DOI:10.1016/j.neuroimage.2019.116351.

Riemer, M., Bublatzky, F., Trojan, J. and Alpers, G. W. (2015). Defensive activation during the rubber hand illusion: ownership versus proprioceptive drift, Biol. Psychol. 109, 86-92. DOI:10.1016/j.biopsycho.2015.04.011.

Riemer, M., Wolbers, T. and Kuehn, E. (2019). Preserved multisensory body representations in advanced age, Sci. Rep. 9, 2663. DOI:10.1038/s41598-019-39270-7.

Rock, I. and Victor, J. (1964). Vision and touch: an experimentally created conflict between the two senses, Science 143, 594-596. DOI:10.1126/science.143.3606.594.

Rognini, G., Sengül, A., Aspell, J. E., Salomon, R., Bleuler, H. and Blanke, O. (2013). Visuotactile integration and body ownership during self-generated action, Eur. J. Neurosci. 37, 1120-1129. DOI:10.1111/ejn.12128.

Rohde, M., Di Luca, M. and Ernst, M. O. (2011). The rubber hand illusion: feeling of ownership and proprioceptive drift do not go hand in hand, PLOS ONE 6, e21659. DOI:10.1371/journal. pone.0021659. 
Romano, D., Pfeiffer, C., Maravita, A. and Blanke, O. (2014). Illusory self-identification with an avatar reduces arousal responses to painful stimuli, Behav. Brain Res. 261, 275-281. DOI:10.1016/j.bbr.2013.12.049.

Salomon, R., Lim, M., Pfeiffer, C., Gassert, R. and Blanke, O. (2013). Full body illusion is associated with widespread skin temperature reduction, Front. Behav. Neurosci. 7, 65. DOI:10. 3389/fnbeh.2013.00065.

Samad, M., Chung, A. J. and Shams, L. (2015). Perception of body ownership is driven by Bayesian sensory inference, PLOS ONE 10, e0117178. DOI:10.1371/journal.pone.0117178.

Schröter, H., Frei, L. S., Ulrich, R. and Miller, J. (2009). The auditory redundant signals effect: an influence of number of stimuli or number of percepts? Atten. Percept. Psychophys. 71, 1375-1384. DOI:10.3758/APP.71.6.1375.

Schwartz, S. H. (1992). Universals in the content and structure of values: theoretical advances and empirical tests in 20 countries, Adv. Exp. Soc. Psychol. 25, 1-65. DOI:10.1016/S00652601(08)60281-6.

Schwarz, N. and Oyserman, D. (2001). Asking questions about behavior: cognition, communication, and questionnaire construction, Am. J. Eval. 22, 127-160. DOI:10.1177/ 109821400102200202.

Shore, D. I., Barnes, M. E. and Spence, C. (2006). Temporal aspects of the visuotactile congruency effect, Neurosci. Lett. 392, 96-100. DOI:10.1016/j.neulet.2005.09.001.

Signorell, A., et mult. al. (2020). DescTools: tools for descriptive statistics. R package version 0.99.34 ed. https://cran.r-project.org/package=DescTools.

Singmann, H., Bolker, B., Westfall, J. and Aust, F. (2017). afex: analysis of factorial experiments, version 0.18-0. Retrieved from http://afex.singmann.science/.

Slater, M., Spanlang, B., Sanchez-Vives, M. V. and Blanke, O. (2010). First person experience of body transfer in virtual reality, PLOS ONE 5, e10564. DOI:10.1371/journal.pone.0010564.

Spence, C. (2013). Just how important is spatial coincidence to multisensory integration? Evaluating the spatial rule, Ann. N. Y. Acad. Sci. U. S. A. 1296, 31-49. DOI:10.1111/nyas.12121.

Spence, C., Pavani, F. and Driver, J. (2004). Spatial constraints on visual-tactile cross-modal distractor congruency effects, Cogn. Affect. Behav. Neurosci. 4, 148-169. DOI:10.3758/CABN. 4.2.148.

Swinkels, L. M. J., van Schie, H. T., Veling, H., ter Horst, A. C. and Dijksterhuis, A. (2020a). The self-generated full body illusion is accompanied by impaired detection of somatosensory stimuli, Acta Psychol. 203, 102987. DOI:10.1016/j.actpsy.2019.102987.

Swinkels, L. M. J., Veling, H., Dijksterhuis, A. and van Schie, H. T. (2020b). Availability of synchronous information in an additional sensory modality does not enhance the full body illusion, Psychol. Res. In press. DOI:10.1007/s00426-020-01396-z.

Tacikowski, P., Fust, J. and Ehrsson, H. H. (2020a). Fluidity of gender identity induced by illusory body-sex change, Sci. Rep. 10, 14385. DOI:10.1038/s41598-020-71467-z.

Tacikowski, P., Weijs, M. L. and Ehrsson, H. H. (2020b). Perception of our own body influences self-concept and self-incoherence impairs episodic memory, iScience 23, 101429. DOI:10. 1016/j.isci.2020.101429.

Technical Support Group (2019). ButtonBoxes [Online]. Available from: https://tsgdoc.socsci. ru.nl/index.php?title=ButtonBoxes [Accessed 05-05-2020 2020]. 
Thür, C., Roel Lesur, M., Bockisch, C. J., Lopez, C. and Lenggenhager, B. (2019). The tilted self: visuo-graviceptive mismatch in the full-body illusion, Front. Neurol. 10, 436. DOI:10. 3389/fneur.2019.00436.

Tieri, G., Tidoni, E., Pavone, E. F. and Aglioti, S. M. (2015). Mere observation of body discontinuity affects perceived ownership and vicarious agency over a virtual hand, Exp. Brain Res. 233, 1247-1259. DOI:10.1007/s00221-015-4202-3.

Tipper, S. P., Phillips, N., Dancer, C., Lloyd, D., Howard, L. A. and McGlone, F. (2001). Vision influences tactile perception at body sites that cannot be viewed directly, Exp. Brain Res. 139, 160-167. DOI:10.1007/s002210100743.

Tosi, G., Parmar, J., Dhillon, I., Maravita, A. and Iaria, G. (2020). Body illusion and affordances: the influence of body representation on a walking imagery task in virtual reality, Exp. Brain Res. 238, 2125-2136. DOI:10.1007/s00221-020-05874-z.

Tsakiris, M., Haggard, P., Franck, N., Mainy, N. and Sirigu, A. (2005). A specific role for efferent information in self-recognition, Cognition 96, 215-231. DOI:10.1016/j.cognition. 2004.08.002.

Ulrich, R., Miller, J. and Schröter, H. (2007). Testing the race model inequality: an algorithm and computer programs, Behav. Res. Methods 39, 291-302. DOI:10.3758/bf03193160.

van der Hoort, B., Guterstam, A. and Ehrsson, H. H. (2011). Being barbie: the size of one's own body determines the perceived size of the world, PLOS ONE 6, e20195. DOI:10.1371/ journal.pone.0020195.

Zizzo, D. J. (2010). Experimenter demand effects in economic experiments, Exp. Econ. 13, 75-98. DOI:10.1007/s10683-009-9230-z.

Zopf, R., Savage, G. and Williams, M. A. (2010). Crossmodal congruency measures of lateral distance effects on the rubber hand illusion, Neuropsychologia 48, 713-725. DOI:10.1016/ j.neuropsychologia.2009.10.028. 